\title{
INFLUENCE OF SENSORIMOTOR TRAINING ON BALANCE AND PAIN PARAMETERS IN CHILDREN WITH HEMOPHILIA
}

\author{
Abeer R. Ibrahim ${ }^{1}$, Azza M. Atya ${ }^{* 1}$, Al Shimaa R. Azab ${ }^{2}$. \\ ${ }^{1}$ Basic Sciences Department, Faculty Of Physical Therapy, Cairo University, Egypt. \\ ${ }^{3}$ Department of Physical Therapy for Growth and Developmental Disorders in Children and its \\ Surgery, Faculty of Physical Therapy, Cairo University, Egypt.
}

\section{ABSTRACT}

Background and purpose: Children with hemophilia often have intra-articular and intra-muscular bleeding which have a worse impact on postural stability and balance. Therefore, the purpose of this study was to investigate the effect of sensorimotor training on balance and perceived pain in children with hemophilia.

Subjects and Methods: Thirty hemophilic boys mean age (12.06 \pm 1.2$)$ were divided randomly into two equal groups. Control group $(n=15)$ received conventional physical therapy program and study group $(n=15)$ received the same program in addition to sensorimotor training exercises. Baseline assessment included stability indices and perceived pain, was conducted at the beginning of the study and after 8 weeks of treatment program.

Results: There was no significant difference between both groups in the pre-treatment mean values of all measured variables. Significant improvement was observed in the two groups between pre and post treatment measured outcomes. Furthermore, the study group recorded significantly better improvement in balance and pain parameters compared with the control group.

Conclusion: Sensorimotor training could be an excellent supplement to regularly rehabilitation program used for children with hemophilia. It produces positive effects on balance and perceived pain parameters for these patients.

KEY WORDS: Sensorimotor Training, Balance, Pain, Hemophilia.

Address for correspondence: Azza Mohamed Atya, Assistant Professor of physical therapy, Basic Sciences department, Faculty of Physical Therapy, Cairo University, Egypt.

Phone Number: +201005208479, E-Mail: azzaatya73@gmail.com

\begin{tabular}{|l|l|}
\hline \multicolumn{3}{|c|}{ Access this Article online } \\
\hline \multirow{2}{*}{ Quick Response code } & \multicolumn{1}{|c|}{ International Journal of Physiotherapy and Research } \\
& \multicolumn{1}{|c|}{ ISSN 2321- 1822 } \\
www.ijmhr.org/ijpr.html
\end{tabular}

\section{INTRODUCTION}

Hemophilia describes a group of sever congenital coagulation factor deficiencies. It is a lifelong genetic disorder with a prevalence of 1:10,000 birth and that of the most severe form of the disease to be approximately $6 \%$ per $1,00,000$ Population [1]. Depending on the activity of coagulant factor VIII or IX in blood, hemophilia may be labeled as severe $(<1 \%$ of normal), moderate (1-4\%) or mild (5-25\%) [2]. Hemophilia is characterized by recurrent haema- rthroses inside the less muscular padding joints as knees, elbows and ankles joints [3].

Because immature cartilage is even more susceptible to spontaneous bleeding, most of hemophilic children experience repeated episodes of hemorrhage into their joints [4]. Intra-articular bleeding of synovial joints has a direct destructive effect on cartilage which triggers the joint degenerative process [5] and impact on the total motor development of children and youngsters [6]. 
Patients with hemophilia often used to have a sedentary lifestyle because of repeated uncontrollable hemarthroses with subsequent synovitis and arthropathy, which is the major complication of hemophilia. The chronic characters of hemophilic patients include musculoskeletal pain, muscle imbalance [7], reduced joint range of motion [8], and articular instability [9].

Balance performance is complex and multidimensional, involving not only the ability to maintain a static stance, but also the ability to maintain equilibrium under a range of dynamic conditions[10]. Few of studies have addressed the impact of musculoskeletal disorders in hemophiliacs on balance impairment or fall. Results showed that patients with hemophilia displayed a worse balance and proprioception compared with healthy normal subjects [10-13].Authors hypothesized that because proprioception information is transmitted from receptors found in all tissues of the synovial joint, repeated joint bleeding into the target joint could be a contributory cause of impaired proprioception in patients with hemophilia. As the muscular function comprises not only the static control of the joint and the dynamic control of movement, but also the proprioceptive input to the central nervous system (CNS) So further possible mechanism induced proprioception deficit in hemophilic patients may be related to the intramuscular bleeding and lower muscle strength [14, 15].

Conservative physical therapy management of hemophilic patients focused primarily on increasing flexibility and muscle strength with minimum joint stress to reduce risk of trauma and further bleeds[16]. Yet, to date and to our knowledge, there are no studies on balance improvement approaches for children with hemophilia. In the past few years, it has been suggested that sensorimotor training contribute to reflective activation of the deep postural muscles and challenge the sensorimotor system to restore normal control of individual segments during dynamic tasks[17-19]. Although many therapists and clinicians reported successful treatment cases, the exact effect and validity of sensorimotor interventions is still discussed with contradicted results [20-22]. Therefore, this study aimed to investigate the feasibility and efficacy of sensorimotor training on improving balance performance and pain in children with hemophilia.

\section{SUBJECTS AND METHODS}

Subjects: Thirty boys with mild to moderate hemophilia were selected from outpatient clinic, faculty of physical therapy, Abu El-Rish Paediatric Hospital, Cairo University, Egypt. Subjects were assigned randomly to two equal groups (control and study). Children in both groups were clinically and medically stable and their medical management included factor replacement therapy which determined by their hematologist. They participated in this study according to the following inclusive criteria: age 10-14 years, understood and followed verbal commands in both test and training, grade $3+$ muscle strength for lower limb according to Kendall et al. [23].

Exclusive criteria included congenital or acquired skeletal deformities, cardiopulmonary dysfunction, advanced radiographic changes as bone erosions or destruction, bony ankyloses, joint subluxation or epiphyseal fracture, neurological condition affecting lower limbs, children who had bleeding in joints or muscles in the 2 weeks prior to assessment and treatment procedure.

Participants were assigned to two equal groups. Control group $(n=15)$ received a conventional physical therapy program and a study group $(n=15)$, received sensorimotor training in addition to a conventional physical therapy program fig 1. Following baseline assessment, children were allocated to corresponding group using a computer-generated randomized table. Allocation was concealed by random numerical sequence in sealed opaque envelopes. The parents of participants provided a consent form for their children to participate in the study as well as acceptance of the Ethics Committee of the Faculty of Physical Therapy, Cairo University was taken.

Methods: Dynamic balance parameter in the form of overall stability index (OSI), medial/ lateral stability index (MLSI), and anterior/ posterior stability index (APSI) and Perceived pain were evaluated using Biodex stability index 
and self-report faces scale respectively at baseline and after 8 weeks.

Balance measurement: Biodex Stability System (BSS) was used to assess the dynamic balance at the Balance Lab, Faculty of Physical Therapy, Cairo University. The BSS reliability been reported [24]. The BBS was calibrated before each measurement according to the manufacturer's manual. Dynamic balance test of BBS was used. It was performed on stability levels from 6 to 4 . Certain parameters were fed to the device including: child's age, weight, height, and stability level.

Each child in the two groups was instructed to achieve a centered position on the platform once the platform with the two leg stance while grasping the handrails by shifting position of feet until it is easy to keep the cursor centered on the screen grid while standing in a comfortable, upright position .child then was instructed that the platform will now be released. Once centering is achieved on the unstable foot platform and the cursor is in the center of the display target, child was instructed to maintain position and the platform was stabilized. Then child's foot position on the foot platform grid was measured.

Three trials were done before testing for learning. After introducing feet angles and heel coordinates into BBS, the test began, the platform advanced to an unstable state, and then the child was instructed to focus on feedback screen directly in front of him. While both hands at the side of his body and asked to maintain the cursor in the middle of on the screen. The test duration was $30 \mathrm{~s}$ ( $\mathrm{sec}$.). The result was displayed on the screen at the end of each test including OSI, APSI and MLSI. The mean of the three repetitions was determined. A large Values was indicated that the child had balance difficulty and poor neuromuscular response [25].

Pain measurement: Pain intensity was measured using the self-report faces scale. Each children was asked to score their average pain experience using a self-report face pain scale which is valid and reliable and has good psychometric properties [26]. Measurement score was done pre-treatment procedure and after 8 weeks treatment for two groups.
Procedures: Patients in both groups received conventional physical therapy program for three sessions/ week on alternate days for 8 weeks in succession. This programme included the following:

$-1^{\text {st }}$ and $2^{\text {nd }}$ weeks: Gentle stretching exercises for tight muscles include biceps brachii, hamstrings, anterior tibial group and calf muscles bilaterally. It was done for $20 \mathrm{~s}$ stretch followed by $20 \mathrm{~s}$ relaxation and repeated five times per session for each muscle, $15 \mathrm{~min}$.

$-3^{\text {rd }}, 4^{\text {th }}$ and $5^{\text {th }}$ weeks (in addition): strengthening exercises in from of Isometric contraction for quadriceps, hamstrings, anterior tibial group, calf muscles triceps and biceps. It was done $5 \mathrm{~s}$ contraction then relaxed for another $5 \mathrm{~s}$ for five times per session[27].

$-6^{\text {th }}, 7^{\text {th }}$ and $8^{\text {th }}$ weeks (in addition): Aerobic exercise in the form of 30 min treadmill training which consisting of 5 min of warm up exercise consisting of stretching exercise and walking forth and back then treadmill training was begun. Treadmill speed was done for all children in both groups as $75 \%$ of their comfortable speed during over ground walking and zero degree inclination for $20 \mathrm{~min}[28,29]$. The child was instructed to stop walking immediately if he felt pain, fainting, or shortness of breath. Finally, cooling down exercises for 5 min involving light stretch and walking inside the room were performed.

Patients in the study group received sensorimotor training program in addition to the conventional programme. Each child in the study group exercised through three phases: static, dynamic, and functional phase. Within each stage child' progress through exercises program in different postures, bases of support, and challenges to their center of gravity [30]. Sensorimotor training was performed 3 times per week for 8 weeks.

Data analysis: The primary outcome measure was pain during rest, stability indices (OSI, MLSI, APSI). The statistical analyses were conducted using the SPSS software, Version 24 (SPSS Inc., Chicago, Illinois). Statistical significance was set at $p<0.05$. Data were summarized as mean and standard deviation. Test for normal data distribution was conducted using KolmogorovSmirnov test. 
Fig. 1: Flow chart of the 35 participants through the study.
Table 1:

Sensorimotor training program.
Two factor mixed model analysis of variance( ANOVA) was used to detect treatment effect between baseline and post-treatment values over a period of eight weeks (within groups variables) and for intra group comparison (control or study ). Between-groups effect sizes for all variables were calculated using the Cohen $d$ coefficient. An effect size $>0.8$ was considered large, around 0.5 moderate, and $<0.2$ small. All out outcome analysis were conducted according to intension to treatment principle.

\section{RESULT}

Thirty-five patients with hemophilia were assessed for compatibility with the eligibility criteria. Thirty met the eligibility criteria and agreed to participate in the study. Patients were randomly allocated to either control or study
Assessed for eligibility ( $n=35$ )

Excluded $(n=5)$

- Not meeting inclusion criteria $(n=3)$

- Declined to participate $(n=2)$ 
Comparison of the pre- and post-treatment values of the OSI score for each group revealed a significant reduction in OSI score in both group after treatment $(p<0.05)$. Post-treatment measurement comparison showed a significant difference between groups in favour of the study group $(p=0.001)$ figure 2 .

Fig. 2: Mean and SD of OSI between groups.

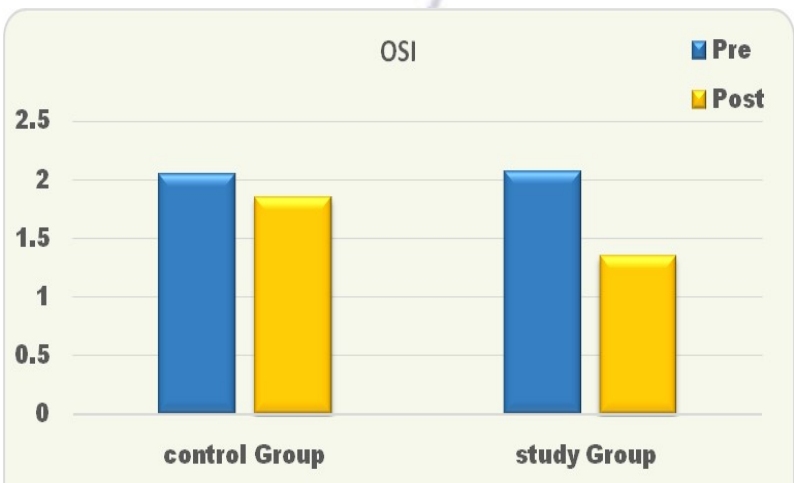

The APSI and MLSI were significantly improved in both group post-intervention when comparing with the base line measurement table 3 . Between groups analysis showed that there were a significant reduction in APSI and MLSI in favour of the study group at the end of treatment.

Data for pain scores at pre-and post-intervention for both groups are reported in Table 3 . There was no significance difference $(p>0.05)$ in pre intervention pain intensity between the groups $(p=0.2)$. Pain intensity was significantly lower in post intervention compared to pre- intervention within two group. At postintervention, the pain felt in the study group was less severe $(p=0.009)$ than the pain felt by control group.

Table 3: Mean (SD) for main outcomes for each group, mean difference $(S D)$ within groups, mean difference and effect size between groups.

\begin{tabular}{|c|c|c|c|c|c|c|c|c|}
\hline \multirow{2}{*}{ Variables } & \multicolumn{4}{|c|}{ Group } & \multicolumn{2}{c|}{$\begin{array}{c}\text { Difference within } \\
\text { group }\end{array}$} & \multicolumn{2}{|c|}{$\begin{array}{c}\text { Difference between } \\
\text { groups }\end{array}$} \\
\cline { 2 - 9 } & Before treatment & \multicolumn{2}{|c|}{ After treatment } & control & study & $\begin{array}{c}\text { Control- } \\
\text { study }\end{array}$ & Effect size \\
\cline { 2 - 8 } & control & study & control & Study & & & \\
\hline OSI & $2.05(0.22)$ & $2.07(0.18)$ & $1.85(0.23)$ & $1.35(0.15)$ & $\begin{array}{c}1.33^{\ddagger} \\
(0.97)\end{array}$ & $\begin{array}{c}1.86^{\ddagger} \\
(0.99)\end{array}$ & $0.50^{*}$ & 0.63 \\
\hline APS & $2.1(0.25)$ & $1.98(0.33)$ & $1.79(0.23)$ & $1.23(0.18)$ & $\begin{array}{c}0.19^{\ddagger} \\
(0.24)\end{array}$ & $\begin{array}{c}0.71^{\ddagger} \\
(0.15)\end{array}$ & $0.56^{*}$ & 0.65 \\
\hline MLS & $1.44(0.18)$ & $1.40(0.24)$ & $1.32(0.23)$ & $0.82(0.17)$ & $\begin{array}{c}0.2^{\ddagger} \\
(0.18)\end{array}$ & $\begin{array}{c}0.75^{\ddagger} \\
(0.3)\end{array}$ & $0.49^{*}$ & 0.6 \\
\hline PAIN & $6.6(1.3)$ & $7.2(1.4)$ & $5.86(1.18)$ & $4.73(1.03)$ & $\begin{array}{c}0.11^{\ddagger} \\
(0.18)\end{array}$ & $\begin{array}{c}0.58^{\ddagger} \\
(0.28)\end{array}$ & $1.13^{*}$ & 0.2 \\
\hline
\end{tabular}

‡ Significance within group * Significance between groups

\section{DISCUSSION}

The purpose of this study was to investigate the effect of sensorimotor training on balance and perceived pain parameters in children with hemophilia. The results of the study demonstrate that children in the study group who had performed sensorimotor training showed greater significant improvement in post- training mean values of stability indices (OSI, APSI, MLSI) and pain severity compared with children in control group.

The difference of the post training values between the two groups could be attributed to improved posture and movement perception by the effect of sensorimotor training. Hertel suggested that sensorimotor training has the ability to enhance balance ability and improve of postural stability by improving mechanoreceptor function, which could lead to restoration of neuromuscular control of the joints [31]. This restoration of the neuromuscular feedback control may be a key factor that causes a positive outcomes in the study group.

Furthermore, it has been estimated that sensorimotor training could help to increase coordination between posture muscle groups and trigger the response to sensorial information. Also during sensorimotor training, patients progressed through exercises in different postures, base of support, challenges to their center of gravity, with and without visual input. So, each exercise program elicits automatic and reflexive muscular stabilization demanding the child to maintain postural control under a variety of situations [32, 33].

The study group showed more significant reduction of pain than the control group. The increased rate of joints and muscle bleeding in hemophilic patients is considered a real problem which lead to swelling, muscle spasm and pain in the affected area[34] .

Balance training rarely have been considered as a part of treatment program for children with hemophilia. Consequently, sensorimotor rehabilitation techniques for sensory deficiencies through promoting proprioceptive challenge have emerged in recent years and have received growing therapeutic attention [12].

Postural control is a complex skill based totally 
on the interaction of dynamic sensorimotor processes. Afferent information from somatosensory, vestibular and visual systems is incorporated according to the goals of the movement task and the environmental context. Postural stability includes coordination of movement strategies in order to maintain the center of body mass during both self-initiated and externally induced disturbances of equilibrium [35].

The findings of this study were similar to the results of Tsauo et al. who concluded that patients performing a set of eight-week sensorimotor training exercises produced better knee joint proprioception and self-reported functional difficulty score [36]. Also Lephart et al. suggested that sensorimotor training may help to improved quality of postural control by increasing of joint position-and motion perception, which in turn may alleviate postural specific musculoskeletal pain [37]. Moreover Gruber et al. reported that sensory motor training may increase proprioceptive input to the neuromuscular system for processing information of the proprioceptive system more appropriately[20]. Another study by Hwang et al. revealed that sensorimotor training makes patients capable of learning how to adjust muscles, thereby alleviating pain and improving muscle performance [38].

In contrast with the current study, Bruhn et al failed to demonstrat a significant difrence between sensorymotor training and strength training on poature stabilization [39]. Also our finding inconsistent with the finding of recent systematic review that appraise the effect of sensorimotor training on functional and balance in total knee replacement patients.The results showed that sensorimotor training induces equivalent improvement between intervention and control group [40]. Where this review based on six studies, generalization of findings should be made with caution, and was only feasible in patients with knee OA undergoing primary total knee replacement.

\section{CONCLUSION}

In this study, sensorimotor training appears to improve balance and pain intensity after eight weeks of application in children with hemophilia.
This study may provide rational for adding sensorimotor training as part of a multifaceted exercise program for patients with hemophilia, particularly if there is a history balance impairment.

\section{ACKNOWLEDGEMENTS}

The authors would like to express their appreciation to all patients who participated in the study and their parents for their cooperation.

\section{Conflicts of interest: None}

\section{REFERENCES}

[1]. Islam S, Morshed AA, Khan ZJ. Hemophilia in Children. Journal of Paediatric Surgeons of Bangladesh. 2016;6(2):54-63.

[2]. Ljung R. Prophylactic therapy in haemophilia. Blood reviews. 2009;23(6):267-74.

[3]. Collins P. Treatment of acquired hemophilia A. Journal of Thrombosis and Haemostasis. 2007;5(5):893900.

[4]. Van den Berg H, Fischer K, Mauser Bunschoten E, Beek F, Roosendaal G, Van der Bom J, et al. Long term outcome of individualized prophylactic treatment of children with severe haemophilia. British journal of haematology. 2001;112(3):561-5.

[5]. Dunn A. Pathophysiology, diagnosis and prevention of arthropathy in patients with haemophilia. Haemophilia. 2011;17(4):571-8.

[6]. Kleijn P, Mauser Bunschoten E, Fischer K, Smit C, Holtslag $H$, Veenhof $C$. Evidence for and cost effectiveness of physiotherapy in haemophilia: a Dutch perspective. Haemophilia. 2016.

[7]. Beeton K, Alltree J, Cornwall J. Rehabilitation of muscle dysfunction in haemophilia. Haemophilia. 2001;(24):1-12.

[8]. Soucie JM, Cianfrini C, Janco RL, Kulkarni R, Hambleton J, Evatt B, et al. Joint range-of-motion limitations among young males with hemophilia: prevalence and risk factors. Blood. 2004;103(7):2467-73.

[9]. Engelbert RH, Plantinga $M$, Van der Net J, Van Genderen FR, Van den Berg MH, Helders PJ, et al. Aerobic capacity in children with hemophilia. The Journal of pediatrics. 2008;152(6):833-8.

[10].Fearn M, Hill K, Williams S, Mudge L, Walsh C, McCarthy $P$, et al. Balance dysfunction in adults with haemophilia. Haemophilia. 2010;16(4):606-14.

[11]. Gallach J, Querol F, Gonzalez L, Pardo A, Aznar J. Posturographic analysis of balance control in patients with haemophilic arthropathy. Haemophilia. 2008;14(2):329-35.

[12]. Hilberg T, Herbsleb M, Gabriel H, Jeschke D, Schramm W. Proprioception and isometric muscular strength in haemophilic subjects. Haemophilia. 2001; 7(6):582-8. 
[13]. Souza F, McLaughlin P, Pereira R, Minuque N, Mello $M$, Siqueira $C$, et al. The effects of repetitive haemarthrosis on postural balance in children with haemophilia. Haemophilia. 2013;19(4):e212-e7.

[14]. Falk B, Portal S, Tiktinsky R, Weinstein Y, Constantini $\mathrm{N}$, Martinowitz U. Anaerobic power and muscle strength in young hemophilia patients. Medicine and science in sports and exercise. 2000;32(1):527.

[15]. Chen L, Wu R, Zhang G. Affects of proprioception training on bleeding frequency in target joints of low extremities in children with moderate-severe hemophilia. Chinese Journal of Rehabilitation Medicine. 2010;4(25):340-342.

[16]. Heijnen L, Buzzard BB, editors. The role of physical therapy and rehabilitation in the management of hemophilia in developing countries. Seminars in thrombosis and hemostasis 2005.

[17]. McCoy SW, Jirikowic T, Price R, Ciol MA, Hsu L-Y, Dellon B, et al. Virtual Sensorimotor Balance Training for Children With Fetal Alcohol Spectrum Disorders: Feasibility Study. Physical therapy. 2015;95(11):1569-81.

[18]. McCaskey MA, Schuster-Amft C, Wirth B, de Bruin ED. Effects of postural specific sensorimotor training in patients with chronic low back pain: study protocol for randomised controlled trial. Trials. 2015 Dec 15;16(1):571.

[19]. Page P. Sensorimotor training: A "global" approach for balance training. Journal of Bodywork and Movement Therapies. 2006;10(1):77-84.

[20]. Gruber M, Gollhofer A. Impact of sensorimotor training on the rate of force development and neural activation. European journal of applied physiology. 2004;92(1-2):98-105.

[21]. Ludwig O, Fröhlich M, Schmitt E. Therapy of poor posture in adolescents: Sensorimotor training increases the effectiveness of strength training to reduce increased anterior pelvic tilt. Cogent Medicine. 2016;3(1):12-31.

[22]. Hupperets MD, Verhagen EA, van Mechelen W. Effect of sensorimotor training on morphological, neurophysiological and functional characteristics of the ankle. Sports medicine. 2009;39(7):591-605.

[23]. Kendall FP, Kendall FP. Muscles : testing and function with posture and pain. Baltimore, MD: Lippincott Williams \& Wilkins; 2005.

[24]. Schmitz R, Arnold B. Intertester and Intratester Reliability of a Dynamic Balance Protocol Using the Biodex Stability System. JOURNAL OF SPORT REHABILITATION. 1998;7(2):95-101.

[25]. Pereira HM, Campos TFd, Santos MB, Cardoso JR, Garcia MdC, Cohen M. Influence of knee position on the postural stability index registered by the Biodex Stability System. GAIPOS Gait \& Posture. 2008;28(4):668-72.

[26]. Bieri D, Reeve RA, Champion GD, Addicoat L, Ziegler $J B$. The faces pain scale for the self-assessment of the severity of pain experienced by children: Development, initial validation, and preliminary investi- gation for ratio scale properties. Pain. 1990; 41(2):139-50.

[27]. Zaky LA, Hassan WF. Effect of partial weight bearing program on functional ability and quadriceps muscle performance in hemophilic knee arthritis. EJMHG Egyptian Journal of Medical Human Genetics. 2013;14(4):413-8.

[28]. Combs SA, Dugan EL, Passmore M, Riesner C, Whipker $D$, Yingling $E$, et al. Balance, Balance Confidence, and Health-Related Quality of Life in Persons With Chronic Stroke After Body Weight-Supported Treadmill Training. YAPMR Archives of Physical Medicine and Rehabilitation. 2010;91(12):1914-9.

[29]. El-Meniawy GH, Kamal HM, Elshemy SA. Role of treadmill training versus suspension therapy on balance in children with Down syndrome. Egyptian Journal of Medical Human Genetics. 2012;13(1):37-43.

[30].Pavlu D, Novosadova K. Contribution to the objectivization of the method of sensorimotor training stimulation according to Janda and Vavrova with regard to evidence-based-practice. Rehabil Phys Med. 2001;8(4):178-81.

[31]. Hertel J. Sensorimotor deficits with ankle sprains and chronic ankle instability. Clinics in sports medicine. 2008;27(3):353-70.

[32]. Solomonow M, Krogsgaard M. Sensorimotor control of knee stability. A review. Scandinavian journal of medicine \& science in sports. 2001;11(2):6480.

[33]. Kapreli E, Athanasopoulos S, Papathanasiou M, Van Hecke P, Kelekis D, Peeters R, et al. Lower limb sensorimotor network: issues of somatotopy and overlap. Cortex. 2007;43(2):219-32.

[34]. Blanchette V, Key NS, Ljung L, Manco Johnson M, Berg $\mathrm{H}$, Srivastava A. Definitions in hemophilia: communication from the SSC of the ISTH. Journal of Thrombosis and Haemostasis. 2014;12(11):19359.

[35]. Horak FB. Postural orientation and equilibrium: what do we need to know about neural control of balance to prevent falls? Age and ageing. 2006;35(suppl 2):ii7-ii11.

[36]. Tsauo J-Y, Cheng P-F, Yang R-S. The effects of sensorimotor training on knee proprioception and function for patients with knee osteoarthritis: a preliminary report. Clinical Rehabilitation. 2008;22(5):448-57.

[37]. Lephart S, Fu F. Proprioception and neuromuscular control in joint stability. Human Kinetics, Champaign. Rozzi S, Yuktanandana P, Pincivero D, Lephart SM. 2000:375-383.

[38]. Hwang JA, Bae SH, Do Kim G, Kim KY. The effects of sensorimotor training on anticipatory postural adjustment of the trunk in chronic low back pain patients. Journal of physical therapy science. 2013;25(9):1189-92.

[39]. Bruhn S, Kullmann N, Gollhofer A. The effects of a sensorimotor training and a strength training on postural stabilisation, maximum isometric contraction and jump performance. International journal of sports medicine. 2004;25(01):56-60. 
[40]. Moutzouri M, Gleeson N, Billis E, Panoutsopoulou I, Gliatis J. What is the effect of sensori-motor training on functional outcome and balance performance of patients' undergoing TKR? A systematic review. Physiotherapy. 2016;102(2):136-44.

How to cite this article:

Abeer R. Ibrahim, Azza M. Atya, Al Shimaa R. Azab. INFLUENCE OF SENSORIMOTOR TRAINING ON BALANCE AND PAIN PARAMETERS IN CHILDREN WITH HEMOPHILIA. Int J Physiother Res 2017;5(2):1912-1919. DOI: $10.16965 /$ ijpr.2017.101 